\title{
Solar Prominences - An Intriguing Phenomenon
}

\author{
Einar Tandberg-Hanssen
}

Received: 4 November 2010 / Accepted: 16 December 2010 / Published online: 19 January 2011

(C) Springer Science+Business Media B.V. 2011

\begin{abstract}
The article starts with an autobiographical account, where the author relates how his several study-trips abroad gradually led him to the study of solar physics in general, and prominences particularly.

The article then treats the historical development of prominence research, from the "speculative" period, before the introduction of photography and spectroscopy around 1860. These techniques led to a new understanding of the nature of prominences as "hot clouds in the solar atmosphere". However, it was only after about 1960, when the magnetic field in prominences could be measured, that the more complete picture of prominences could be understood, a view greatly helped by space missions, the result of which was the realization that the solar atmosphere is crisscrossed by electric currents that lead to magnetic flux tubes of nearly all imaginable sizes.

The article ends with a discussion of the disparition-brusque phenomenon, and the closely related coronal mass ejections.
\end{abstract}

\section{Autobiographical Introduction}

Since the first reported observation of "a cloud in the Sun's atmosphere", numerous interpretations for this strange phenomenon have been proposed. Today we have come a long way in understanding the physics of prominences, but it has often been a difficult road, and there are still unresolved problems for new generations of solar physicists to explore.

My own interest in solar physics probably started when I - as a graduate student in the early post-World-War-II years - helped build the Solar Observatory of the University of Oslo, and aimed the telescope, with an $\mathrm{H} \alpha$ filter, at the Sun, and admired the beautiful prominences. However, not much became of these early observations, but I may consider this effort as a training period. I studied under Rosseland, so my interest therefore also went

E. Tandberg-Hanssen is retired.

E. Tandberg-Hanssen $(\bowtie)$

Center for Space Plasma and Aeronomic Research, University of Alabama in Huntsville, Huntsville, AL 35899, USA

e-mail: einar.tandberg@gmail.com 
to stellar physics, and astrophysics in general. I have always enjoyed this broad interest in astrophysics, and the role of magnetic fields in stellar research particularly intrigued me. In the 1950s solar magnetism was a hot subject, and when I returned to Oslo after some study years abroad, still under Rosseland's guidance, Eberhart Jensen - a fellow student, who was always a little ahead of me, and who, deservedly, later would follow Rosseland as Director of our Astrophysical Institute - and I wrote a paper on the role of magnetic fields in the heat exchange between cool prominences and the hot corona (Rosseland, Jensen, and Tandberg-Hanssen, 1958).

In 1951 I left Oslo to spend the academic year in Paris, at the Institut d'Astrophysique. There I had the good fortune of meeting a number of scientists, who all, directly or indirectly, helped me decide that solar physics should be my field: Chalonge, Dollfus, Martres, Michard, Pecker, Schatzman, Steinberg. The year in Paris was very fruitful for me, and I would later go back many times in the 1980s and 1990s to work with - or at least discuss with - the "next generation" of solar physicists: Bommier, Démoulin, Hénoux, Koutchmy, Lantos, Leroy, Malherbe, Mein (P. and N.), Mouradian, Sahal, Simon, SoruEscaut, Schmieder, Vial, and I have probably unintentionally overlooked some. We had many interesting discussions on the dynamics of prominences as seen in $\mathrm{H} \alpha$ at the Meudon solar tower, and compared these with C IV observations of the UVSP instrument on board the Solar Maximum Mission ( $\mathrm{PhD}$ thesis of Malherbe, 1989).

We discussed the nature of active prominences and flares, and the question was raised whether other stars also would have flares. To try to answer this question, Kourganoff and I went to Provence, to L'Observatoire de Haute Provence near Manosque, and spent several weeks looking at what we thought would be promising flare-star objects. We did not observe any flares, and abandoned that research project.

I mention the list of solar scientists I met in Paris, because I think it is very important to keep up a good working relationship with colleagues in one's field, and it indicates who some of the solar physicists were, with whom I enjoyed a good working relationship in the waning years of the 20th century. I visited France: Paris and in particular Provence (that my wife and I had fallen in love with in the early 1950s) every year around the IAU meetings of FAGS (Federation of Astronomical and Geophysical Analysis Services), and it was always a pleasure to meet my French colleagues. I wrote two books for my daughters about my visits to France.

The academic year 1952/53 I spent at California Institute of Technology in Pasadena, California, where we experienced the exciting time when Baade, Feynman and Zwicky reshaped our view of cosmic physics. I worked with Greenstein, who taught me how to calculate the abundance of atomic species in the Sun, from spectrographic observations, and one of my first scientific papers was titled The Abundance of Beryllium in the Sun (Greenstein and Tandberg-Hanssen, 1954), where we found a striking excess of beryllium as compared with lithium; a phenomenon that greatly intrigued me, and further sharpened my interest in solar physics.

My third, and final, "study trip" abroad took place during the spring of 1954, which I spent in Cambridge, England. The field of radio astronomy was quickly developing, and I worked in Martin Ryle's group, which had spear-headed this branch of solar physics after World War II. I acquired an appreciation for the possibility of probing the solar atmosphere in the radio-frequency domain.

After a couple of years at the University of Oslo, where I lectured in solar physics, I moved in 1957 to Boulder, Colorado, where I would remain at the High Altitude Observatory until 1974. Those were exciting years: I studied nearly all types of solar activity, concentrating on prominences, but since some types of active prominences and flares often 
Figure 1 Portrait of Einar Tandberg-Hanssen.

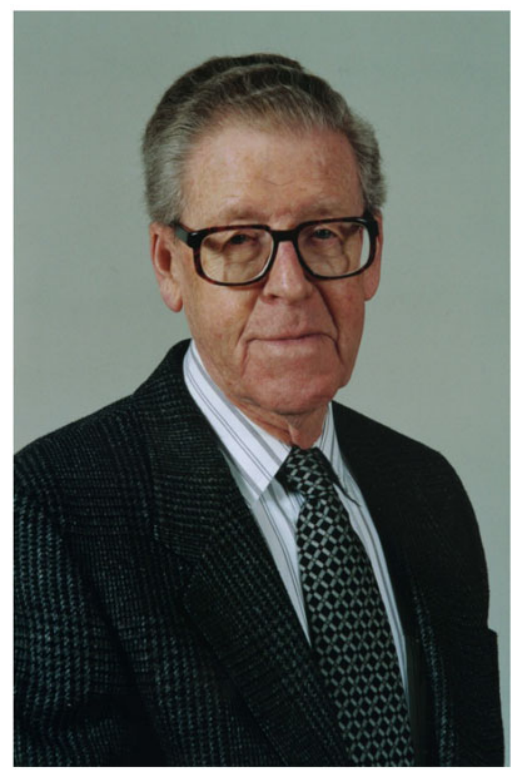

occur simultaneously, I also made considerable efforts to understand the flare phenomenon. Zirin and I studied spectra of active and quiescent prominences, and developed a classification system (Zirin and Tandberg-Hanssen, 1960), where we also considered flares. Toward the end of my stay I felt I knew enough about prominences to write a textbook about them, entitled Solar Prominences (Tandberg-Hanssen, 1974).

When my programs in Boulder came to an end in 1974, I was offered a position in Huntsville, Alabama, at NASA's Marshall Space Flight Center, where I remained until retirement roughly 20 year later (see Figure 1 for a recent portrait). Those were the exciting years with the Solar Maximum Mission, which resulted in a vast increase in our knowledge of solar prominences. The result was that I considered my textbook on prominences from 1974 to be outdated, and toward the end of my NASA years I completely rewrote the textbook, and produced a new version called The Nature of Solar Prominences (TandbergHanssen, 1995).

I enjoyed and shared my curiosity for prominences with many colleagues during meetings on prominence physics, i.e. Symposium on Solar Prominences, September 1971, at Capri, Italy; IAU Colloquium No. 44 in Oslo, Norway, August 1978, organized by Jensen; a Solar Maximum Mission workshop "Coronal and Prominence Plasmas" at GSFC in 1986 (see Figure 2), organized by Poland; the IAU Colloquium No. 117 in September 1989, at Hvar, Croatia, organized by Ruždjak and Tandberg-Hanssen (see Figure 3); the IAU Colloquium No. 167 "New Perspectives on Solar Prominences", April 1997, in Aussois, France, organized by Webb, Rust and Schmieder (see Figure 4).

\section{A Little Prominence History}

Early in my studies I spent some time on the history of prominences (see Tandberg-Hanssen, 1998), and I was struck by the fact that it was not until the eclipse in 1851, observed in Norway and Sweden, that a proper solar interpretation of the prominence phenomenon emerged 


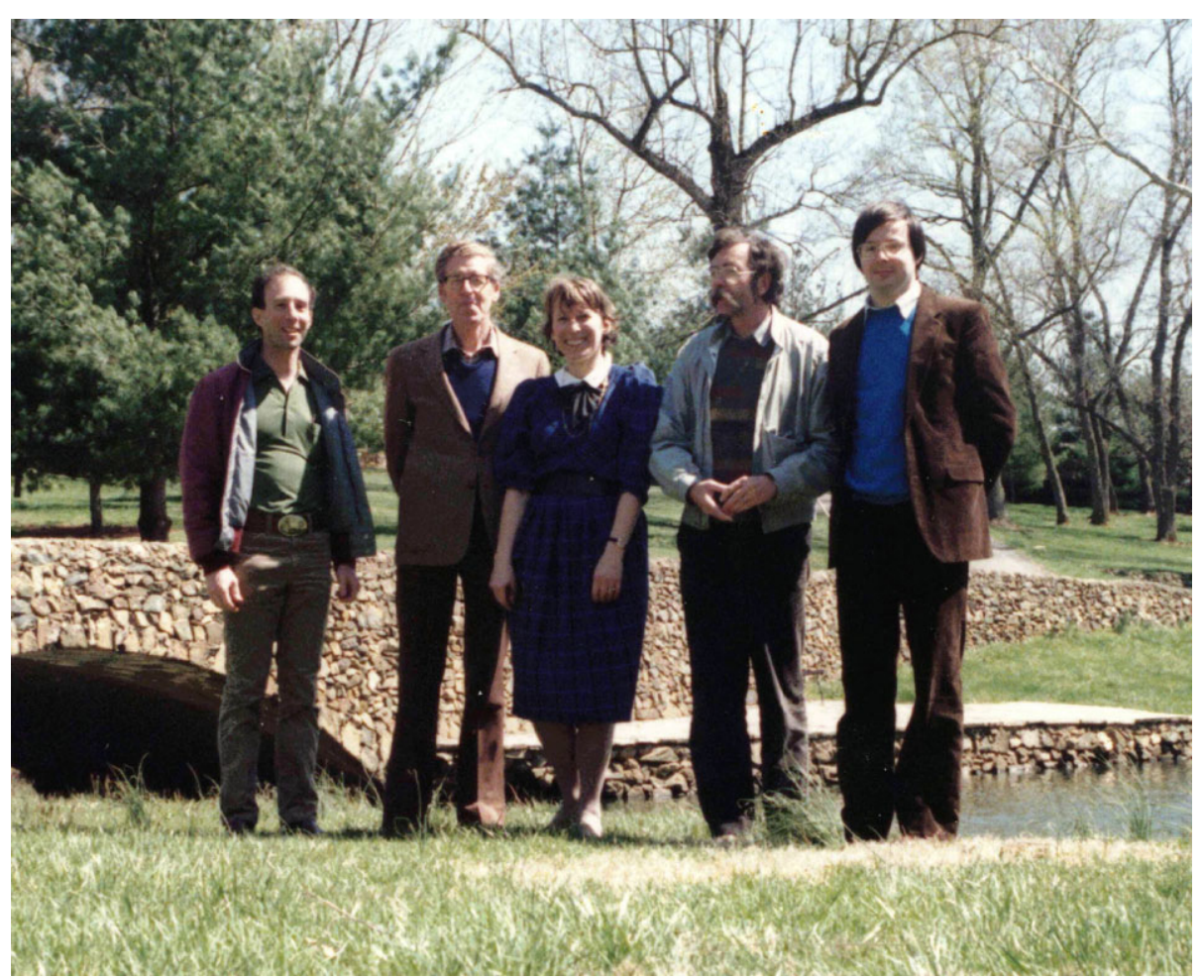

Figure 2 Photo taken at the SMM Workshop in Airlie in 1986 (from left to right: Art Poland, Einar Tandberg-Hanssen, Brigitte Schmieder, Guy Simon, Jean-Marie Malherbe).

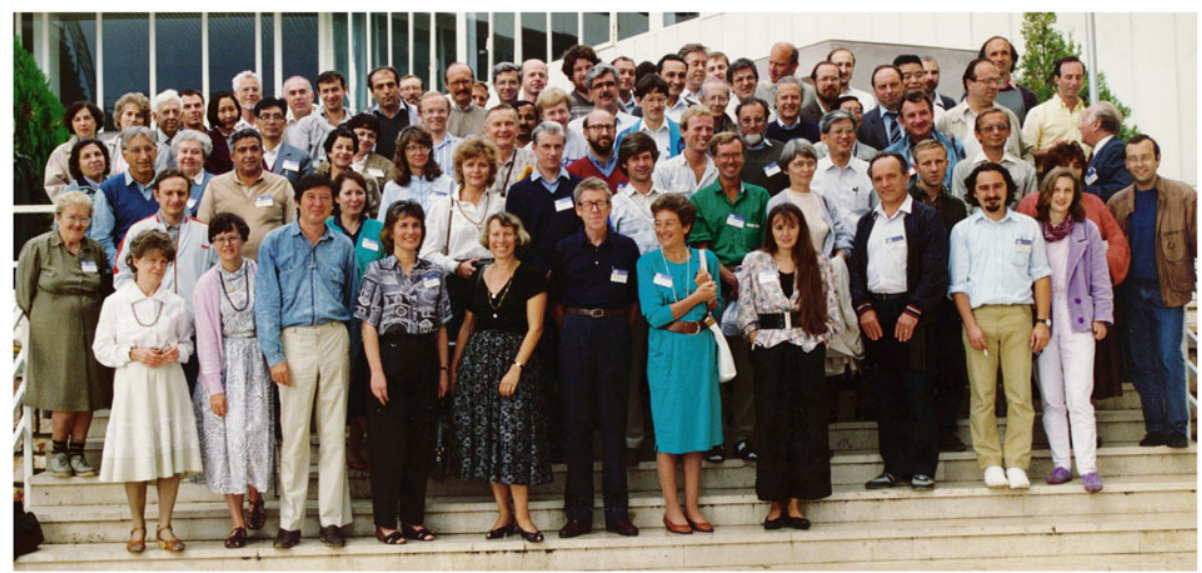

Figure 3 Participants at IAU Colloq. No. 117 "Dynamics of Quiscent Prominences", Hvar, Croatia, 1989.

(see Secchi, 1875). Earlier interpretations had included "lunar clouds" or a "hole in the Moon". Then with the introduction of photography at the eclipse in Spain in 1860, and 


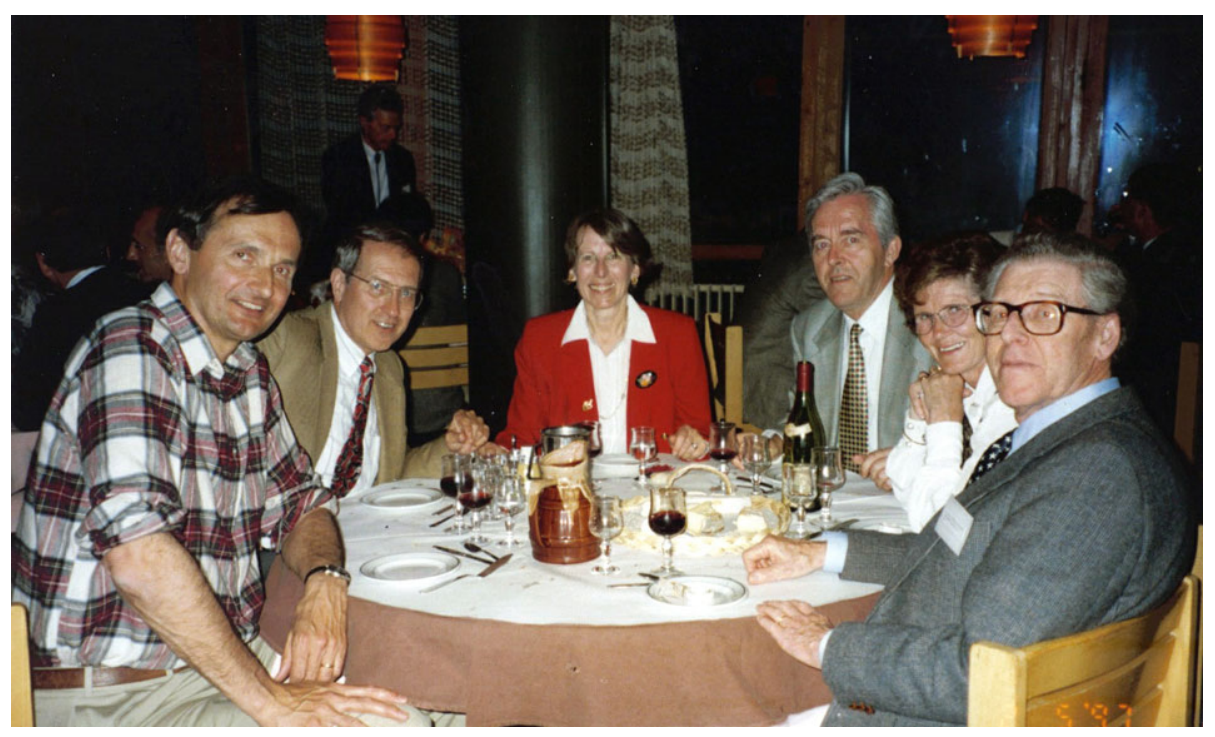

Figure 4 Conference Dinner in Aussois, France, at the IAU Symposium 167 in April 1997. From left to right: Peter Foukal, Dave Rust, Brigitte Schmieder, Oddbjorn Engvold and his wife, and Einar Tandberg-Hanssen.

spectrography at the eclipse in India and Malacca in 1868 one realized that prominences are masses of solar glowing gas (Secchi, 1875).

For convenience I would like to consider three epochs in the history of prominence research:

i) the speculative period, before 1860 ,

ii) the spectrographic period, $1860-1960$,

iii) the polarimetric period, after 1960 .

The dates are approximate, and probably the only merit in presenting these epochs lies in emphasizing the importance of being able to analyze spectral lines and, later, to discover the all-important electric currents and associated magnetic fields in the prominence plasma.

In his truly remarkable book Le Soleil Father Secchi described the speculative period, and led us into the next. The use of spectroscopy was such a potent tool, that in $1875 \mathrm{Secchi}$ started his chapter on prominences with the statement "The phenomenon of prominences is now so well known by everybody that it may seem unnecessary to retrace the history of their discovery". It makes one humble to realize that 135 years later, this "well-known phenomenon" still poses some puzzling questions for solar physicists. Nevertheless, considerable progress has been made in our understanding of the nature of solar prominences. Already in 1906, Schwarzschild (1906) published the results of the first systematic photometric measurements of spectral lines in prominences, using the data from the 1905 eclipse.

In the 1890 s spectroheliographs became available, and prominences could be studied on the disk as absorption features (Hale and Ellerman, 1903; Deslandres, 1910). And then came Lyot's invention of the coronagraph (Lyot, 1936), and it became possible to observe limb prominences at any time, not only during eclipses.

The actual magnetograph measurements of the longitudinal magnetic field via the Zeeman effect in spectral lines from prominences ushered in our third epoch, the polarimetry 
period (Zirin and Severny, 1961; Ioshpa, 1962). By then it had already become clear that magnetic fields were likely to play a role in the physics of prominences. Babcock and Babcock (1955) pointed out that quiescent prominences were to be found along the neutral line between the two opposite polarities in bi-polar magnetic regions. A little later Hyder (1964) used the theory of resonance polarization and impact polarization to estimate the longitudinal magnetic field in prominences from studies of the observed linear polarization of prominence emission lines.

The progress that followed is due to the analysis, via the Zeeman effect, of several sets of data from coronagraph-magnetograph combinations especially built for prominence research. At the High Altitude Observatory we mostly used the $\mathrm{H} \alpha$ line, but at times also the Na I, D3 line (Rust, 1966; Harvey and Tandberg-Hanssen, 1968; Malville, 1968).

One of the most important data sets then came from the use of the Hanle effect and was due to the pioneering work at Pic-du-Midi Observatory, with its new instrumentation (Ratier, 1975), and at Meudon Observatory, where the theoretical foundation and an innovative data analysis took place (Sahal-Brechot, Bommier, and Leroy, 1977; Leroy, 1979; Bommier and Sahal-Brechot, 1979).

The advent of the space era brought us the series of Orbiting Solar Observatories (OSOs), Skylab 1973, Solar Maximum Mission 1980 - 1989, Spacelab missions, the Yohkoh satellite 1991 - 2001,TRACE, and SOHO 1996 - . More recently we have the Japanese satellite Hinode 2005 -, STEREO 2007 - , and the Solar Dynamic Observatory 2010 - . Instrumentations on board several of these spacecraft have made it possible to push prominence observations into the UV, EUV, and even the X-ray domain.

While the space-research efforts pushed observations toward shorter wavelengths, the radio astronomers provided us with information on the prominence plasma from studies in the $\mathrm{mm}$ to $\mathrm{dm}$ wavelength region; see e.g. Chiuderi Drago (1990). Disk observations of prominences in their filament channels can be used to assess the physical conditions in the chromospheres around these objects (Gary, 1986; Hiei et al., 1986; Kundu, 1986).

\section{Concentration on Prominences}

During my years in Boulder, I more and more concentrated my research in the area of prominences and their environments. There are many forms of prominences, and already the famous Italian Secchi, in his truly remarkable book (Secchi, 1875), showed that while some prominences are long-lasting, quiescent objects, others - called active (Secchi called them eruptive) - display sudden changes, and are - generally speaking - short-lived.

Many attempts have been made to classify the different types of prominences: some classifications are morphological, some spectroscopic. For many years the most widely known and used morphological classification was that due to Pettit $(1925,1932)$. He divided prominences into five classes, as shown in Table 1.

Pettit's class 5 refers to the classical quiescent prominences that take the form of enormous sheets standing more or less vertically in the solar atmosphere.

With the advent of spectroscopic studies of prominences, it became clear that different classes of prominences showed differences in the ratios of certain spectral-line intensities, and based on this a new way of prominence classification became available. The first modern spectral classification is due to Waldmeier $(1949,1951,1961)$, who used the $b$ lines of Mg I ( $b_{1}$ at $5184 \AA, b_{2}$ at $5172 \AA$ and $b_{4}$ at $5167 \AA$ ) and compared their intensities with the intensity of the $b_{3}$ line of Fe II at $5167 \AA$; see Table 2 . 
Table 1 Pettit's classification (adopted from Tandberg-Hanssen, 1995).

Table 2 Waldmeier's classification (adopted from Tandberg-Hanssen, 1995).

Table 3

Zirin - Tandberg-Hanssen classification (adopted from Tandberg-Hanssen, 1995).

\begin{tabular}{|c|c|c|}
\hline Class & Name & Description \\
\hline 1 & Active & $\begin{array}{l}\text { Material seems to be streaming into nearby active } \\
\text { center (like sunspots) }\end{array}$ \\
\hline 2 & Eruptive & $\begin{array}{l}\text { The whole prominence ascends with uniform ve- } \\
\text { locity (of several hundred } \mathrm{km} \mathrm{s}^{-1} \text { often). The ve- } \\
\text { locity may at times suddenly increase }\end{array}$ \\
\hline 3 & Sunspot & $\begin{array}{l}\text { These are found near sunspots and take the shape } \\
\text { of "water in a fountain" or loops }\end{array}$ \\
\hline 4 & Tornado & $\begin{array}{l}\text { A vertical spiral structure gives these prominences } \\
\text { the appearance of a closely wound rope or whirling } \\
\text { column }\end{array}$ \\
\hline 5 & Quiescent & $\begin{array}{l}\text { Large prominence masses which show only minor } \\
\text { changes over periods of hours or days }\end{array}$ \\
\hline Class & & Criteria \\
\hline I & & $I\left(b_{3}\right)<I\left(b_{4}\right)$ \\
\hline II & & $I\left(b_{3}\right)=I\left(b_{4}\right)$ \\
\hline III & & $I\left(b_{4}\right)<I\left(b_{3}\right) \leq I\left(b_{2}\right)$ \\
\hline IV & & $I\left(b_{2}\right)<I\left(b_{3}\right) \leq I\left(b_{1}\right)$ \\
\hline $\mathrm{V}$ & & $I\left(b_{3}\right)>I\left(b_{1}\right)$ \\
\hline
\end{tabular}

\begin{tabular}{|c|c|c|}
\hline Class & Criteria & Objects \\
\hline I & $\begin{array}{l}I(\mathrm{He} \mathrm{I}, 4026) \ll I(\mathrm{Sr} \mathrm{II}, 4078) \\
I(\mathrm{He} \mathrm{I}, 4713) \ll I(\mathrm{Ti} \text { II }, 4572) \\
I(\text { He II, 4686) } \ll I(\mathrm{He} \mathrm{I}, 4713)\end{array}$ & Low Chromosphere \\
\hline II & 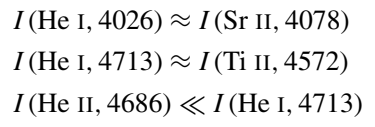 & $\begin{array}{l}\text { Middle Chromosphere } \\
\text { (around } h=1500 \mathrm{~km} \text { ) } \\
\text { Quiescent Prominences }\end{array}$ \\
\hline III & $\begin{array}{l}I(\mathrm{He} \mathrm{I}, 4026) \gg I(\mathrm{Sr} \mathrm{II}, 4078) \\
I(\mathrm{He} \mathrm{I}, 4713) \gg I(\mathrm{Ti} \mathrm{II}, 4572) \\
I(\mathrm{He} \mathrm{II}, 4686) \approx I(\mathrm{He} \mathrm{I}, 4713)\end{array}$ & $\begin{array}{l}\text { High Chromosphere } \\
\text { (spicules) } \\
\text { Active Prominences Flares }\end{array}$ \\
\hline IV & $\begin{array}{l}\text { Presence of } \\
\text { Fe X, 6374, } \\
\text { Fe XIV, 5303, } \\
\text { Ca XIII, 4086, or } \\
\text { Ca XV, } 5694\end{array}$ & $\begin{array}{l}\text { Coronal Enhancements } \\
\text { Coronal Condensations }\end{array}$ \\
\hline
\end{tabular}

Waldmeier found that flares generally fall in classes IV and V, while prominences mostly belong to class III, with a few cases in classes I and II. This classification distinguishes between flares and prominences, but it does not significantly sub-divide prominences. Another classification, not only of prominences, but including other solar atmospheric objects, is due to Zirin and Tandberg-Hanssen (1960), see Table 3. 
Zirin and I worked on this classification at The High Altitude Observatory in the late 1950s. It is based on the idea of a multi-component model, i.e. on the idea that flares and active prominences consist of different regions. Some regions are cool, less than $10^{4} \mathrm{~K}$, with strong hydrogen and metal-line emission, while the He emissions are weak. Other regions are composed of hotter plasma with temperatures significantly in excess of $10^{4} \mathrm{~K}$, giving rise to strong $\mathrm{He}$ and faint metal-line emissions. A comparison between the classification lines gives a measure of which regions dominate a given object. As seen in Table 3, such a comparison furnishes a convenient way of classifying different aspects of solar activity, and of comparing it with the chromosphere (the quiet Sun). Flares and active prominences fall in the same class III, characteristic of high chromospheres, indicating that, spectroscopically, active prominences are more like flares than like quiescent prominences. The latter fall in class II and may be considered similar to the middle chromospheres in excitation. Extremely quiescent prominences may even border on class I.

The spectroscopic distinction between flares and active prominences, which is brought out in Waldmeier's classification, is not present in the original Zirin-Tandberg-Hanssen classification. To remedy this, the intensity of certain metal lines that behave differently in flares and in active prominences was introduced as classification criteria (Tandberg-Hanssen, 1963). The distinction can be made by using lines of Fe II, which are strong in flares, and comparing them with lines of Ba II and especially Ti II, which characterize spectra of prominences. Since the other classification lines in the easily observable blue-green part of the spectrum lie around $4600 \AA$, the $4584 \AA$ line of Fe II (multiplet 38 ) and the Ti II line at $4572 \AA$ (multiplet 82 ) were chosen as our flare/active prominence discriminators. The ratio $M=I$ (Fe II, 4584) $/ I$ (Ti II, 4572), where $I$ (Fe II, 4584) and $I$ (Ti II, 4572) are the intensities of the two lines, respectively, will then classify an active limb event as a prominence when $M$ is less than one, and as a flare when $M$ is equal to or greater than one. It was not obvious to me why Ti II lines should be more prominent in active prominences, like loops and surges, than in flares, and I never pursued this question.

Considerable progress had been made in our understanding of the physical properties of prominences at the time of the Hvar meeting (IAU Colloquium No. 117, 1989, see Figure 3), and a model of prominences was developed at that meeting, the so-called Hvar Model (Engvold et al., 1990), and a table of prominence characteristics was proposed by Jensen and Wiik (1990). The Hvar reference model is still valid, but I would encourage the readers of this article to read the more recent review of the plasma physics in prominences, written by Labrosse et al. (2010).

\section{The Importance of Magnetic Fields}

As mentioned in Section 2 the first measurements of the longitudinal component of the magnetic field in prominences were accomplished in 1961. Early measurements, all using the Zeeman effect, are summarized by Tandberg-Hanssen (1974) and Leroy (1979). By using more sophisticated instrumentation, all four Stokes parameters were subsequently measured and the vector field determined. With the use of the Hanle effect a new technique was developed to measure the total (vector) field, and Leroy (1985) summarized the results.

For quiescent prominences the absolute field strength, $|\mathbf{B}|$, is generally found in the range from a few gauss to 10 gauss, occasionally reaching 20 or 30 gauss. While there is no unanimity, several authors find that the field strength is slightly higher in the upper parts of quiescent prominences. Furthermore, there seems to be a weak correlation with the solar cycle: the prominence field is somewhat stronger at solar maximum than at minimum, an effect first pointed out by Harvey (1969). 
a

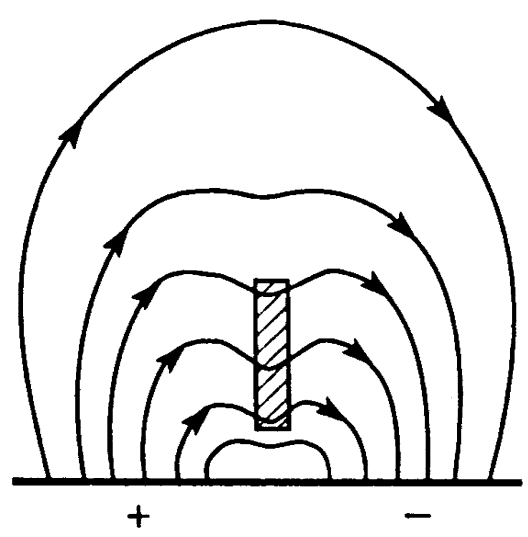

b

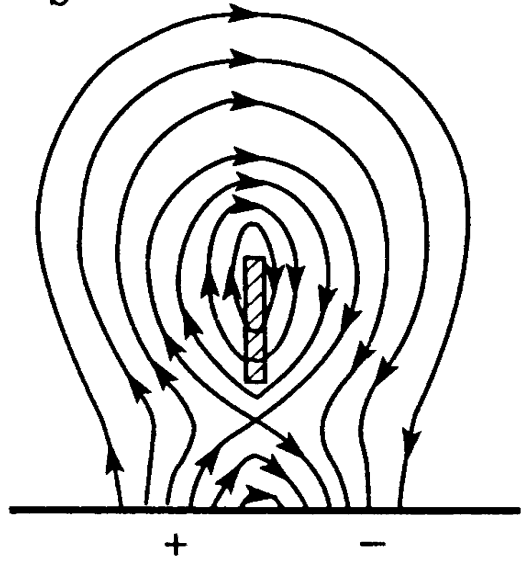

Figure 5 Two possible magnetic field topologies with embedded prominence. (a) Normal polarity; (b) inverse polarity (adopted from Tandberg-Hanssen, 1995).

Of great importance for our understanding of prominence physics is the question of how the magnetic field is oriented in and around the prominence body. In the simplest case of a quiescent object, visualized as a plasma sheet standing vertically in the solar corona above the magnetic neutral line, the magnetic field emanates in one polarity region, enters the prominence, exits from the other side, and proceeds to an opposite polarity region. However, most indications are that the field vector $\mathbf{B}$ is not perpendicular to the prominence sheet. Measurements show that the angle $\alpha$ between the vector $\mathbf{B}$ and the long axis of the prominence is quite small (Tandberg-Hanssen and Anzer, 1970; Kim et al., 1990). With Leroy, Bommier, and Sahal-Brechot (1983, 1984), who studied the problem in considerable detail, we indicate that $\alpha$ has a strong maximum around 25 degrees. This small value of $\alpha$ must be taken into consideration when one examines the role of the magnetic field on the stability of prominences, and how the field supports the prominence plasma.

From an observational point of view there are two main field configurations found in and around quiescent prominences. The first, shown schematically in Figure 5(a), is one associated with prominences where the apparent polarity of the field across the prominence sheet is the same as the polarity of the underlying photospheric field. We refer to these prominences as having a normal polarity field, N-type prominences. One of the early investigations of such prominences is due to Kippenhahn and Schlüter (1957), and we often refer to these prominences as being of the Kippenhahn - Schlüter type.

The other type of field configuration comes from the prominence model initially proposed by Kuperus and Tandberg-Hanssen (1967), and developed by Kuperus and Raadu (1974). This configuration is shown in Figure 5(b) and is referred to as the inverse polarity field, I-type prominences. Leroy, Bommier, and Sahal-Brechot (1984) have extensively studied both $\mathrm{N}$ - and I-type prominences. As a rule, I-type objects seem to be more numerous than N-types, seem to be taller, and seem to be typical of polar crown prominences. N-type prominences are lower than $30000 \mathrm{~km}$.

While the magnetic field in quiescent prominences has been the subject of numerous investigations and, therefore, is well known, until recently less has been known about the field in active prominences. Good data have now been accumulated, and the field in the various 
types of active prominences is reasonably well understood. One should bear in mind that the first measurements made of magnetic fields in prominences (Zirin and Severny, 1961) pertained to active objects, and the early work of Ioshpa (1968) and Harvey (1969) studied the fields in different types of active prominences. The field strength is often considerably greater than in quiescent prominences. Koutchmy and Zirker (1990) found evidence of very strong fields in thin horizontal threads of a low-lying active-region filament. While the field between the threads was smaller than 150 gauss, the field in the threads showed values up to 1000 gauss.

One of the outstanding results from the major solar space-research programs of the 1980s and 1990s is the realization that the solar atmosphere is completely crisscrossed by electric currents that lead to magnetic flux tubes of nearly all imaginable sizes, ranging from small loops in and around plages to gigantic arches that span large distances between different active regions in which their feet are anchored. Some of these flux tubes form the skeletons of different kinds of prominences and constitute their fine structure. For a recent review of the magnetic field in prominences, see Mackay et al. (2010).

\section{Prominence Motions}

Apart from the magnetic field no other parameter is more important for an understanding of the physics of prominences than their motions. This is because of the important forces that are generated when the ionized prominence plasma flows through magnetic fields. It has been known for a long time that the plasma in even the most quiescent prominences shows considerable motion. On high-resolution $\mathrm{H} \alpha$ photographs one observes that the material is concentrated in thin ropes of diameter less than $300 \mathrm{~km}$ (Dunn, 1960, see Figure 6), and from movies one gets the impression that the plasma is slowly either streaming down or rising up these more or less vertical ropes (Dunn, 1965; Engvold, 1976; Anzer, 1979; Cui et al., 1985). This question has been revisited with the recent movies of hedgerow prominences provided by the Japanese satellite Hinode (Kosugi et al., 2007; Schmieder et al., 2010; Chae, 2010; see Figure 6).

Horizontal motions, $V(\mathrm{H})$, are also of great importance for the development of quiescent prominences. Such motions were recorded, e.g., by Mein et al. (1989) who recorded the velocity as $\mathrm{H} \alpha$ blue shift in a prominence above the limb and found $V(\mathrm{H})=15 \mathrm{~km} \mathrm{~s}^{-1}$. Kulidzanishvili (1989) found velocities around $10 \mathrm{~km} \mathrm{~s}^{-1}$, but with considerable spread, and stressed the importance of considering a dynamic equilibrium for quiescent prominences. Schmieder (1989) has reviewed $V(\mathrm{H})$ measurements pertaining not only to $\mathrm{H} \alpha$, but to lines of Mg II (Vial et al., 1979) and "hotter" lines from C IV and Si IX (Athay, Jones, and Zirin, 1985; Lites et al., 1976; Engvold, Tandberg-Hanssen, and Reickmann, 1985).

The importance of these horizontal flows in and around filaments may also be evidenced from analysis of center-to-limb observations of the mass motions, and the magnetic field geometries may be deduced from the dynamic behavior of the chromospheric plasma (Malherbe et al., 1983) or of the transition region plasma (Athay, Jones, and Zirin, 1985). The flows in prominences have been reviewed in recent years by Labrosse et al. (2010).

Another important streaming motion of the prominence plasma may be observed near sunspots. Initially, quiescent prominences form in pre-existing plage regions that may contain sunspots. As a result, we often observe a quiescent prominence, one end of which interacts with a sunspot in such a way that material is streaming out of the prominence and down into the sunspot (Martin, 1973). In this case the velocity is much higher than in the case of the slowly downward streaming motions referred to above. Due to the mass 

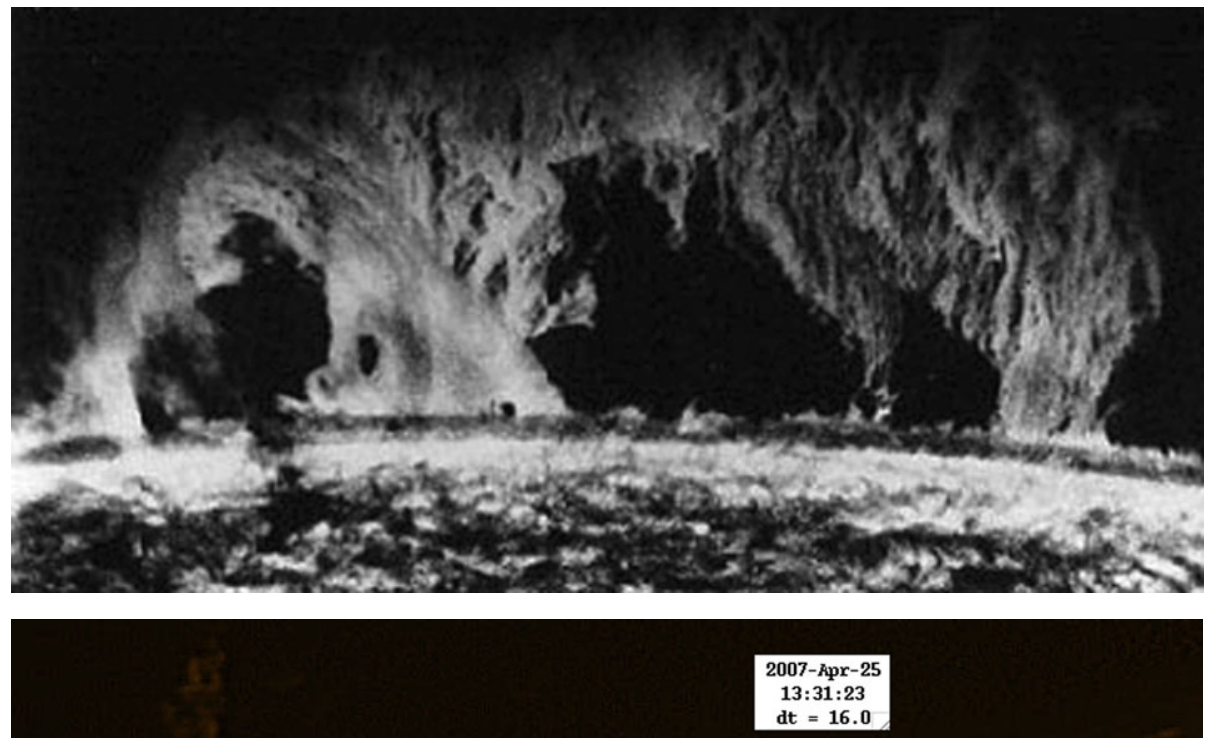

$13: 31: 23$

$\mathrm{dt}=16.0$

Figure 6 (Upper panel) Hedgerow prominence observed at Big Bear Solar Observatory in H $\alpha$ in 1970; (lower panel) prominence observed in H $\alpha$ by Hinode/SOT on 25 April 2007 (courtesy B. Schmieder). 
losses involved, the quiescent prominences that interact with active regions strongly call for a dynamic model. The activation of an active-region filament, be it increased internal motion or a genuine disparition brusque (see later), normally precedes a flare, and hence furnishes a good flare precursor (Martin, 1980; de Jager and Svestka, 1985; Kaastra, 1985; Tandberg-Hanssen and Emslie, 1988).

At times prominences are subjected to external disturbances that result in increased motions or turbulence of the prominence plasma. The severity of the perturbations may range from a slight temporary "activation", manifested by enhanced internal motions, to disturbances of such magnitude that the prominence is completely destroyed. The disturbing agents emanate from flares, growing sunspots, or changes in the general magnetic field structure.

\section{The Disparition-Brusque Phenomenon}

A gross velocity of particular interest results when a quiescent prominence undergoes a sudden disappearance due to an ascending motion. To distinguish it from other types of disappearances, we often employ the French term disparition brusque. The cause is generally not a flare-induced activation (Raadu et al., 1987). Prior to a disparition brusque the prominence plasma exhibits increased random velocities of 30 to $50 \mathrm{~km}$ per second, and then the whole prominence, or most of it, starts to ascend with increasing velocity, which will attain values of several hundred kilometers per second. Closely associated with the disparition-brusque phenomenon are the fascinating changes that occur in the coronal structure called coronal mass ejection (CME). From an observational point of view a coronal mass ejection may be defined as a change in the coronal structure that occurs on times scales up to several hours and involves the appearance of new bright features seen in the K-corona (Munro et al., 1979; Hundhausen et al., 1984). To the extent that the "new bright features" may be due to erupting prominence material, a study of coronal mass ejections forms part of prominence research, particularly as it applies to the disparition-brusque phenomenon.

During the later years of my stay at The High Altitude Observatory in Colorado, I became particularly interested in disparitions brusques and coronal mass ejections, and I had many discussions with my colleague B.C. Low, who developed a comprehensive model for CMEs (Low 1993, 1994). In addition to the outward velocity of the erupting material, the disparition-brusque phase is characterized also by a most interesting spiraling motion and helical structure of the prominence plasma. Early observations are due, e.g., to Severny and Khoklova (1953), Zirin (1968), Slonim (1969), and Anzer and Tandberg-Hanssen (1970). Later, more detailed discussions on the helical structure in eruptive prominences are due to, e.g. Moore (1988), and Rompolt (1990). As the helical flux tube erupts, its magnetic energy should decrease. Moore (1988) considered such a twisted flux tube to find the dependence of its energy on the field expansion and untwisting, and then evaluated the decrease in energy.

My interest in prominence physics has made me reasonably familiar with the mathematical tools necessary to interpret the data. These tools are first and foremost spectroscopy, based on atomic physics, and magneto-hydrodynamics, incorporating transport phenomena. However, I cannot leave this section without mentioning another tool, developed during the last several decades. Few areas of applied mathematical physics have seen a more impressive growth than the model building by computer simulations based on massive numerical calculations, and I have often discussed this development with S.T. Wu, at the University of Alabama in Huntsville, one of the pioneers in this field. The numerical simulation methods are very powerful and can provide new insight into the physical processes taking place under conditions found in solar plasmas (see e.g. Wu et al., 1988). Some recent simulations of 
prominence eruptions and CMEs are due to work by Forbes et al. (2006), and Aulanier et al. (2010).

It is interesting that the coronagraphs on SOHO track a large number of CMEs, one to five per day, depending on the phase of the solar cycle. Fifteen per cent are due to filament eruptions alone, while $59 \%$ involve the eruption of a prominence according to Subramanian and Dere (2001).

\section{Conclusion}

The nature of prominences has undoubtedly been my favorite research topic. Since their discovery 150 years ago, they have been observed with larger and larger ground-based telescopes, as well as from space, thereby extending the wavelength range of observation to include EUV, X-rays and the radio domain.

Theoretical approaches using MHD or radiative-transfer models for prominences have allowed us to make progress in the understanding of the physical conditions in this cool plasma embedded in the hot corona. The instability of prominences initiates coronal mass ejections, which travel through the heliosphere towards the Earth within the solar wind. The CMEs may collide with our atmosphere and thereby become part of the new developing science of "Space Weather".

Acknowledgements I would like to express my gratitude to the Memoir Committee for the honor of being elected to write this memoir. It has been fun to relive the exciting years from the last century, when we realized the importance of the presence of magnetic fields in astronomical bodies.

My thanks go also to the referee, Brigitte Schmieder, who made constructive suggestions to improve the manuscript.

Hinode is a Japanese mission developed and launched by ISAS/JAXA, with NAOJ as domestic partner and NASA and STFC (UK) as international partners. It is operated by these agencies in co-operation with ESA and NSC (Norway).

\section{References}

Anzer, U.: 1979, In: Jensen, E., Maltby, P., Orrall, F.Q. (eds.) Physics of Solar Prominences, IAU Colloq. 44, 322.

Anzer, U., Tandberg-Hanssen, E.: 1970, Solar Phys. 11, 61.

Athay, R.G., Jones, H.P., Zirin, H.: 1985, Astrophys. J. 288, 363.

Aulanier, G., Török, T., Démoulin, P., DeLuca, E.E.: 2010, Astrophys. J. 708, 314.

Babcock, H.D., Babcock, H.W.: 1955, Astrophys. J. 121, 349.

Bommier, V., Sahal-Brechot, S.: 1979, In: Jensen, E., Maltby, P., Orrall, F.Q. (eds.) Physics of Solar Prominences, IAU Colloq. 44, 87.

Chae, J.: 2010, Astrophys. J. 714, 618.

Chiuderi Drago, F.: 1990, In: Ruzdjak, V., Tandberg-Hanssen, E. (eds.) Dynamics of Quiescent Prominences, IAU Colloq. No. 117, Lecture Notes in Phys. 363, Springer, Berlin, 70.

Cui, L.-S., Hu, J., Ji, G.-P., Ni, X.-B., Huang, Y.-R., Fang, C.: 1985, Chin. Astron. Astrophys. $9,49$.

de Jager, C., Svestka, Z.: 1985, Solar Phys. 100, 435.

Deslandres, H.: 1910, Ann. Obs. Paris-Meudon 4(1), 1.

Dunn, R.: 1960, Ph.D. Thesis, Harvard Univ.

Dunn, R.: 1965, Sacramento Peak Obs. Contr. Report \#87.

Engvold, O.: 1976, Solar Phys. 49, 283.

Engvold, O., Tandberg-Hanssen, E., Reickmann, E.: 1985, Solar Phys. 96, 35.

Engvold, O., Hirayama, J.L., Leroy, J.-L., Priest, E., Tandberg-Hanssen, E.: 1990, In: Ruzdjak, V., TanbergHanssen, E. (eds.) Lecture Notes in Physics, 297.

Forbes, T.G., Linker, J.A., Chen, J., Cid, C., Kota, J., Lee, M.A., Mann, G., Miki, Z., Potgieter, M.S., Schmidt, J.M., et al.: 2006, Space Sci. Rev. 123, 251. 
Gary, D.E.: 1986, In: Poland, A.I. (ed.) Coronal and Prominence Plasmas, NASA Conf. Publ. $2442,121$.

Greenstein, J.L., Tandberg-Hanssen, E.: 1954, Astrophys. J. 119, 113.

Hale, G.E., Ellerman, F.: 1903, Publ. Yerkes Obs. 3(1), 3.

Harvey, J.W.: 1969, Ph.D. Thesis, Univ. of Colorado.

Harvey, J., Tandberg-Hanssen, E.: 1968, Solar Phys. 3, 316.

Hiei, E., Ishiguro, M., Kosugi, T., Shibasaki, K.: 1986, In: Poland, A.I. (ed.) Coronal and Prominence Plasmas, NASA Conf. Publ. 2442, 109.

Hundhausen, A.J., Sawyer, C.R., House, L., Illing, R.M.E., Wagner, W.J.: 1984, J. Geophys. Res. 89, 2639.

Hyder, C.L.: 1964, Astrophys. J. 140, 817.

Ioshpa, B.A.: 1962, Geomagn. Aeron. 2, 149.

Ioshpa, B.A.: 1968, In: Kiepenheuer, K.O. (ed.) Structure and Development of Solar Active Regions, IAU Symp. 35, Dordrecht, Reidel, 261.

Jensen, E., Wiik, J.E.: 1990, In: Ruzdjak, V., Tanberg-Hanssen, E. (eds.) Lecture Notes in Physics, 298.

Kim, I.S., Klepikov, V.Y., Koutchmy, S., Stepanov, A.I., Stellmacher, G.: 1990, Sov. Astron. Lett. $16,234$.

Kaastra, J.S.: 1985, Ph.D. Thesis, Univ. of Utrecht.

Kippenhahn, R., Schlüter, A.: 1957, Z. Astrophys. 43, 36.

Kosugi, T., Matsuzaki, K., Sakao, T., Shimizu, T., Sone, Y., Tachikawa, S., Hashimoto, T., Minesugi, K., Ohnishi, A., Yamada, T., et al.: 2007, Solar Phys. 243, 3.

Koutchmy, S., Zirker, J.B.: 1990, In: Ruzdjak, V., Tandberg-Hanssen, E. (eds.) Dynamics of Quiescent Prominences, IAU Colloq. No. 117, Lecture Notes in Phys. 363, Springer, Berlin, 242.

Kulidzanishvili, V.I.: 1989, Hvar Obs. Bull. 13(1), 215.

Kundu, M.R.: 1986, In: Poland, A.I. (ed.) Coronal and Prominence Plasmas, NASA Conf. Publ. $2442,117$.

Kuperus, M., Raadu, M.A.: 1974, Astron. Astrophys. 31, 189.

Kuperus, M., Tandberg-Hanssen, E.: 1967, Solar Phys. 2, 39.

Labrosse, N., Heinzel, P., Vial, J.-C., Kucera, T., Parenti, S., Gunar, S., Schmieder, B., Kilper, G.: 2010, Space Sci. Rev. 151, 243.

Leroy, J.-L.: 1979, In: Jensen, E., Maltby, P., Orrall, F.Q. (eds.) Physics of Solar Prominences, IAU Colloq. 44, 56.

Leroy, J.-L.: 1985, In: Hagyard, M.J. (ed.) Measurements of Solar Vector Magnetic Fields, NASA Conf. Publ. 2374, 121.

Leroy, J.-L., Bommier, V., Sahal-Brechot, S.: 1983, Solar Phys. 83, 135.

Leroy, J.-L., Bommier, V., Sahal-Brechot, S.: 1984, Astron. Astrophys. 131, 33.

Lites, B.W., Bruner, E.C., Chipman, E.G., Shine, R.A., Rottman, G.J., White, O.R., Athay, R.G.: 1976, Astrophys. J. Lett. 210, L111.

Low, B.C.: 1993, Astrophys. J. 409, 412.

Low, B.C.: 1994, Phys. Plasmas 1, 1684.

Lyot, B.: 1936, C. R. Acad. Sci. 202, 392.

Mackay, D.H., Karpen, J.T., Ballester, J.L., Schmieder, B., Aulanier, G.: 2010, Space Sci. Rev. $151,333$.

Malherbe, J.M.: 1989, Ph.D. Thesis, Univ. of Paris.

Malherbe, J.M., Simon, G., Mein, P., Mein, N., Schmieder, B., Vial, J.-C.: 1983, Adv. Space Res. $2(11), 53$.

Malville, J.M.: 1968, Solar Phys. 5, 236.

Martin, S.F.: 1973, Solar Phys. 31, 3.

Martin, S.F.: 1980, Solar Phys. 68, 217.

Mein, P., Mein, N., Schmieder, B., Noens, J.-C.: 1989, Hvar Obs. Bull. 13(1), 113.

Moore, R.L.: 1988, Astrophys. J. 324, 1132.

Munro, R.H., Gosling, J.T., Hildner, E., MacQueen, R.M., Poland, A.I., Ross, C.L.: 1979, Solar Phys. 61, 201.

Pettit, E.: 1925, Publ. Yerkes Obs. 3(IV), 205.

Pettit, E.: 1932, Astrophys. J. 76, 9.

Raadu, M.A., Malherbe, J.M., Schmieder, B., Mein, P.: 1987, Solar Phys. 109, 59.

Ratier, G.: 1975, Nouv. Rev. Opt. 6, 149.

Rompolt, B.: 1990, Hvar Obs. Bull. 14(1), 37.

Rosseland, S., Jensen, E., Tandberg-Hanssen, E.: 1958, In: Lehnert, B. (ed.) Electromagnetic Phenomena in Cosmic Physics, IAU Symp. 6, Cambridge Univ. Press, London, 150.

Rust, D.M.: 1966, Ph.D. Thesis, Univ. of Colorado.

Sahal-Brechot, S., Bommier, V., Leroy, J.-L.: 1977, Astron. Astrophys. 59, 223.

Schmieder, B.: 1989, In: Priest, E.R. (ed.) Dynamics and Structure of Quiescent Solar Prominences, Kluwer Acad., Dordrecht, 15.

Schmieder, B., Chandra, R., Berlicki, A., Mein, P.: 2010, Astron. Astrophys. 514, 68.

Secchi, A.: 1875, Le Soleil, Gauthier-Villars, Paris.

Schwarzschild, K.: 1906, Astron. Mitt. Sternwarte Göttingen 13, 1. 
Severny, A.B., Khoklova, V.L.: 1953, Izv. Krym. Astrofiz. Obs. 10, 9.

Slonim, Yu.M.: 1969, Soviet Astron. 13, 450.

Subramanian, P., Dere, K.P.: 2001, Astrophys. J. 561, 372.

Tandberg-Hanssen, E.: 1963, Astrophys. J. 137, 26.

Tandberg-Hanssen, E.: 1974, Solar Prominences, Reidel, Dordrecht.

Tandberg-Hanssen, E.: 1995, The Nature of Solar Prominences, Kluwer Acad., Dordrecht.

Tandberg-Hanssen, E.: 1998, In: The History of Solar Prominence Research, ASP Conference Series 150, 11. Tandberg-Hanssen, E., Anzer, U.: 1970, Solar Phys. 15, 158.

Tandberg-Hanssen, E., Emslie, A.G.: 1988, In: The Physics of Solar Flares, Cambridge Univ. Press, Cambridge, 140 .

Vial, J.-C., Gouttebroze, P., Artzner, G., Lemaire, P.: 1979, Solar Phys. 61, 39.

Waldmeier, M.: 1949, Z. Astrophys. 26, 305.

Waldmeier, M.: 1951, Z. Astrophys. 28, 208.

Waldmeier, M.: 1961, Z. Astrophys. 53, 142.

Wu, S.T., Wang, S., Wang, A.H., Dryer, M.: 1988, Adv. Space Res. 8(11), 221.

Zirin, H.: 1968, In: Ohmann, Y. (ed.) Mass Motions in Solar Flares and Related Phenomena, Nobel Symp 9, Almqvist \& Wiksell, Stockholm, 131.

Zirin, H., Severny, A.B.: 1961, Observatory 81, 155.

Zirin, H., Tandberg-Hanssen, E.: 1960, Astrophys. J. 131, 717. 